\title{
Imaging and Polarimetry of the Dust Shells Around Post-AGB Stars
}

\author{
Tim Gledhill, Indra Bains \\ Dept. of Physical Sciences, University of Hertfordshire, Hatfield, AL10 \\ $9 A B$, England \\ Jeremy Yates \\ University College London, Gower St., London, WC1E 6BT, England
}

\begin{abstract}
We have completed a polarimetric survey of 46 post-AGB stars which indicates that where an extended circumstellar envelope is seen then it is axisymmetric to some degree. This suggests that all postAGB envelopes are axisymmetric and that the responsible mechanism must be ubiquitous.
\end{abstract}

\section{Introduction}

In order to understand how axisymmetry originates in AGB stars and whether it can lead to the observed morphological diversity of planetary nebulae (PN), we need to study the circumstellar envelopes (CSEs) of the intervening post-AGB phase. These dusty CSEs are seen by scattered light at optical and near-IR wavelengths and appear as linearly polarised reflection nebulae. Many postAGB CSEs are compact $\left(<3^{\prime \prime}\right)$ and are often associated with optically bright central stars. This makes them difficult to resolve from the ground, since the CSE is likely to be hidden under the point-spread function (PSF) of the central star. To overcome this problem we have used imaging polarimetry at the UK Infrared Telescope (UKIRT) to probe the CSE structures of a sample of 46 post-AGB stars (see Gledhill et al. 2001 for a description of the technique). The completion of this survey presents the opportunity to investigate the structure of the CSEs in post-AGB stars across a larger, morphologically unbiased, sample.

\section{Imaging Polarimetry Survey}

Imaging polarimetry observations of 46 PPN were made at UKIRT between May 1998 and July 2001. Results for 16 objects are described in Gledhill et al. (2001), the remainder will appear in a forthcoming paper (Gledhill et al. 2002 in prep.). Polarisation is confidently detected in 36 objects, with a further 6 objects having a marginal detection. Only 4 objects appeared to be unpolarised. We have categorised the range of envelope morphologies on the basis of the observed polarisation pattern and polarised flux distribution, into shell, bipolar and coredominated types. In Fig. 1 we show imaging polarimetry results for 2 shell-type 

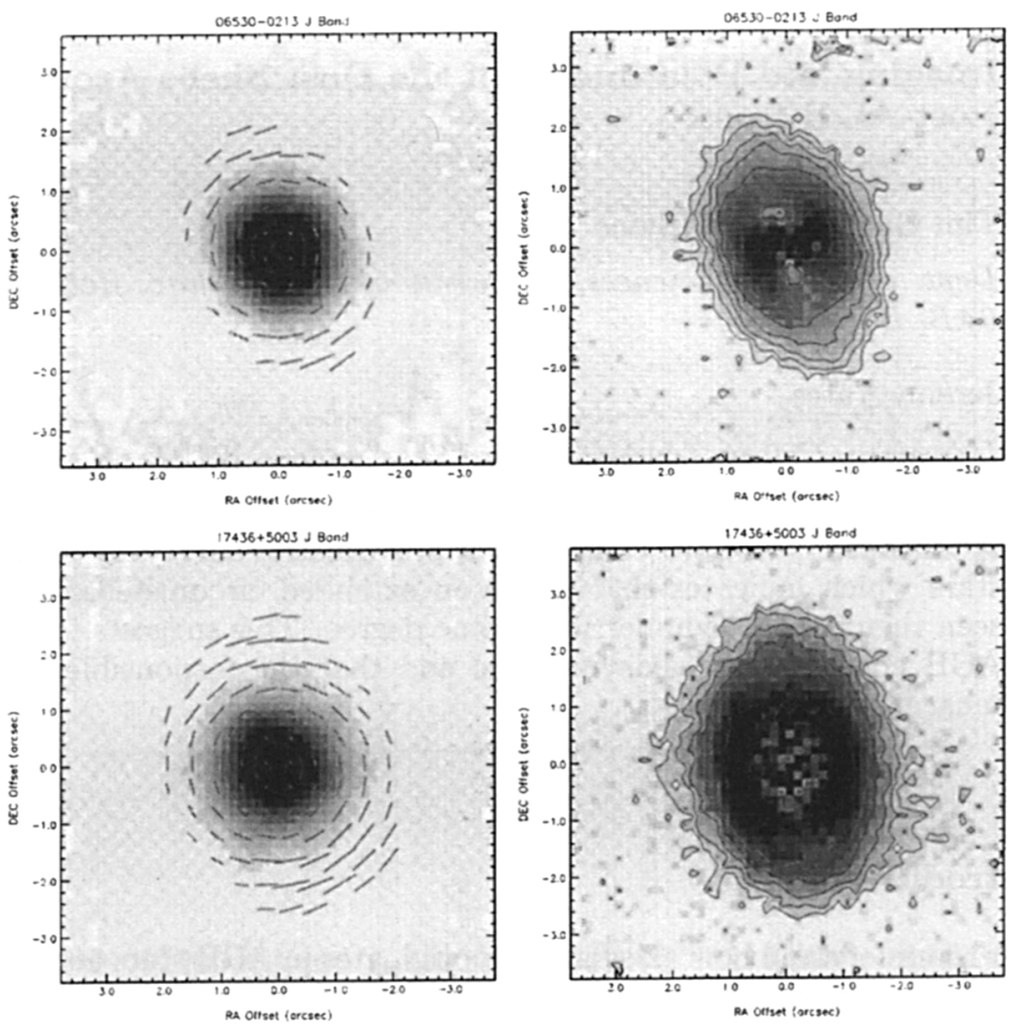

Figure 1. Imaging polarimetry results for 2 post-AGB stars with axisymmetric dust shells. Total flux images (left) are superimposed with polarisation vectors. The polarised flux images (right) show the shell structure.

objects. These types have optically bright central stars with centro-symmetric polarisation patterns indicating that the scattering is occurring in an optically thin envelope or shell. In polarised light, the scattering structure of the envelope is seen, revealing a limb-brightened circumstellar shell.

We find that all the post-AGB CSEs in our sample, where polarisation is detected, show some evidence for axisymmetry suggesting that the mechanism that generates axisymmetry is (i) operational at the end of the AGB or early in the post-AGB phase and (ii) operational in all post-AGB stars. Although binarity is expected to have important effects on the shaping of the post-AGB CSE, our results suggest that a more ubiquitous mechanism, such as magnetic collimation, may be required.

\section{References}

Gledhill T.M., Chrysostomou A., Hough J.H., Yates J.A., 2001, MNRAS, 322, 321 